\title{
Capacidade funcional e qualidade de vida entre sujeitos saudáveis e pacientes com apneia obstrutiva do sono
}

\author{
Functional capacity and quality of life between healthy subjects and patients with \\ obstructive sleep apnea
}

Cadi C. R. Tassinari ${ }^{1}$, Chaiane F. Piccinn ${ }^{2}$, Marco C. Beck ${ }^{3}$, Fabrício Scapini ${ }^{4}$, Luiz C. A. Oliveira ${ }^{5}$, Luis U. Signori6, Antônio M. V. Silva?

\begin{abstract}
RESUMO
Modelo do estudo: Caso-controle. Objetivos do estudo: Comparar a capacidade funcional, parâmetros respiratórios e qualidade de vida entre sujeitos saudáveis e pacientes com apneia obstrutiva do sono. Metodologia: Em 19 pacientes com apneia obstrutiva do sono e 19 sujeitos saudáveis foram avaliadas a qualidade de vida (The Medical Study 36-item Short-Form Health Survey), capacidade funcional (teste de caminhada de seis minutos), função pulmonar (espirometria) e força muscular respiratória (manovacuometria). Resultados: Os pacientes apresentaram declínio na qualidade de vida pelos domínios capacidade funcional, estado geral de saúde e saúde mental. A distância percorrida no teste de caminhada de seis minutos foi menor nos pacientes com apneia obstrutiva do sono. Os parâmetros respiratórios não diferiram entre os grupos. Conclusão: Pacientes com apneia obstrutiva do sono apresentam comprometimento da qualidade de vida e da capacidade funcional.
\end{abstract}

Palavras-chave: Apneia do Sono Tipo Obstrutiva. Aptidão Física. Qualidade de Vida.

\begin{abstract}
Study Model: Case - control. Study Objectives: To compare the functional capacity, respiratory parameters and quality of life between healthy subjects and patients with obstructive sleep apnea. Methodology: 19 patients with obstructive sleep apnea and 19 healthy subjects were evaluated regarding their quality of life (The Medical Study 36-Item Short-Form Health Survey), their functional capacity (six-minute walk test), pulmonary function (spirometry) and their strength respiratory muscle (manovacuometer). Results: Patients showed a decline in the quality of life concerning functional capacity domains, general state of health and mental health. The distance walked in the six-minute walk test was shorter for patients with obstructive sleep apnea. Respiratory parameters did not differ between groups. Conclusion: Patients with obstructive sleep apnea present impairment regarding their quality of life and functional capacity.
\end{abstract}

Keywords: Sleep Apnea, Obstructive. Physical Fitness. Quality of Life.

1. Fisioterapeuta, pós-graduanda em Reabilitação Físico-Motora pela Universidade Federal de Santa Maria (UFSM), Santa Maria, RS, Brasil.

2. Educadora Física e Fisioterapeuta, Mestranda em Distúrbios da Comunicação Humana pela UFSM.

3. Fisioterapeuta, Especialista em Reabilitação Físico-Motora pela UFSM.

4. Médico Otorrinolaringologista, Instituto do Sono de Santa Maria (ISSM), Santa Maria, RS. Doutor em Ciências pela Universidade de São Paulo. Docente no Departamento de Clínica Médica, UFSM.

5. Médico Otorrinolaringologista, ISSM.

6 Fisioterapeuta, Doutor em Ciências da Saúde: Cardiologia pela Fundação Universitária de Cardiologia, Porto Alegre, RS, Brasil. Docente da UFSM.

7 Fisioterapeuta, Doutor em Ciências Biológicas: Fisiologia pela Universidade Federal do Rio Grande do Sul, Porto Alegre, RS, Brasil. Docente da UFSM.
Correspondencia

Prof. Dr. Antônio Marcos Vargas da Silva Rua Sete, 156. Alto da Colina. Bairro

Camobi

CEP: 97110-785-Santa Maria, RS, Brasil.

Recebido em 06/10/2014 Aprovado em 20/10/2015 


\section{Introdução}

A apneia obstrutiva do sono (AOS) é uma patologia de grande prevalência na população adulta em geral, compondo altos índices de morbidade e mortalidade. ${ }^{1}$ Os pacientes apresentam como principais sinais e sintomas, o ronco alto, o sono não reparador e transtornos de comportamento e humor. ${ }^{1}$ A AOS também é descrita como uma condição comum causada por colapso intermitente das vias aéreas durante o sono que resulta em hipóxia repetitiva, despertar noturno, piora na qualidade do sono e sonolência excessiva diurna. ${ }^{2}$ A qualidade de vida pode estar comprometida nesses pacientes, ${ }^{3,4}$ isso ocorre devido aos sintomas diurnos, como a sonolência excessiva, a irritabilidade, a diminuição da concentração e da memória, as quais interferem nas suas atividades sociais. ${ }^{3}$ Esse comprometimento está relacionado com a gravidade do distúrbio do sono. ${ }^{4}$

A capacidade funcional, que é a habilidade que $o$ indivíduo apresenta para realizar, de forma autônoma, as atividades de vida diária (AVD'S), ${ }^{5}$ pode estar afetada nesses pacientes. ${ }^{6}$ De acordo com a literatura, há controvérsias quanto às limitações na capacidade funcional de pacientes com AOS. Estudos prévios ${ }^{7-10}$ demonstraram que a capacidade funcional apresentou-se reduzida em pacientes com diagnóstico de AOS não tratada, 7,8 enquanto que outros estudos ${ }^{9,10,11}$ não confirmaram esses resultados. As disfunções pulmonares também podem estar presentes, pois à má qualidade do sono, resultante do aumento do colapso intermitente das vias aéreas superiores limitam o fluxo inspiratório e diminuem o volume expiratório forçado no primeiro segundo (VEF1). ${ }^{9}$ Além disso, o aumento do volume pulmonar tem efeito estabilizador sobre a via aérea durante o sono em paciente com AOS. ${ }^{10}$ Atualmente, o principal tratamento utilizado é a Pressão Positiva Contínua nas Vias Aéreas (CPAP), que objetiva a normalização do volume pulmonar. ${ }^{11}$

Os efeitos da AOS sobre a capacidade funcional, função pulmonar e qualidade de vida ainda não estão totalmente compreendidos. Segundo estudos citados anteriormente, ${ }^{7-11}$ estes impasses se devem as ausências do controle das comorbidades e dos métodos terapêuticos empregados aos pacientes, e também a falta de comparação com sujeitos saudáveis. Assim, o presente estudo objetiva com- parar a capacidade funcional, função pulmonar, força muscular respiratória e qualidade de vida entre sujeitos saudáveis e pacientes com diagnóstico de AOS.

\section{Materiais e métodos}

Este estudo do tipo caso-controle foi aprovado pelo Comitê de Ética em Pesquisa (CEP) da Universidade Federal de Santa Maria (UFSM), sob o número do CAAE 08798612.0.0000.5346, conforme a Resolução 196/96 do Conselho Nacional de Saúde. Foram estudados 19 pacientes com AOS Grupo apneia obstrutiva do sono (GAOS), diagnosticados pela polissonografia basal de noite inteira no Instituto do Sono de Santa Maria (ISSM), de ambos os gêneros, com idade entre 18 a 65 anos. O grupo controle (GC) foi constituído por 19 sujeitos saudáveis, pareados por gênero e idade, oriundos da comunidade local. Os sujeitos com alguma disfunção cognitiva que pudesse interferir no entendimento das avaliações, com impossibilidade de ler ou assinar o Termo de Consentimento Livre e Esclarecido (TCLE), com diagnóstico de doenças cardíacas, vasculares, metabólicas ou doença pulmonar obstrutiva crônica, tabagistas e com índice de massa corporal (IMC) acima de $30 \mathrm{~kg} / \mathrm{m}^{2}$ não foram incluídos no estudo. Os sujeitos do grupo controle que relataram qualquer tipo de distúrbio do sono avaliado pelo Índice de Qualidade do Sono de Pittsburg (PSQI-BR) e pela Escala de Sonolência de Epworth (ESS) também foram excluídos. Todos os voluntários aceitaram e assinaram o TCLE.

As avaliações dos pacientes do GAOS foram realizadas de acordo com o agendamento da equipe do ISSM. As avaliações do GC ocorreram conforme a disponibilidade dos voluntários. As avaliações foram realizadas no turno da manhã, nas dependências do ISSM e no Laboratório de Reabilitação Físico-Motora da UFSM, entre março de 2013 e abril de 2014. Os sujeitos preencheram uma ficha de identificação e de triagem, nas quais foram registrados dados como: idade, massa corporal, estatura, sinais vitais e saturação periférica de oxigênio $\left(\mathrm{SpO}_{2}\right)$.

Aplicaram-se o PSQI-BR e a ESS para avaliação de questões relacionadas ao sono. O PSQI-BR, validado para a língua portuguesa, ${ }^{12}$ foi utilizado para avaliar a qualidade do sono em relação ao último mês, analisando a combinação de informações quantitativas e qualitativas do sono. A ESS é um 
questionário projetado para avaliar o nível de sonolência durante o dia habitual. A escala é composta por oito itens com típicas situações do dia-a-dia. Cada item pode ser classificado de 0 a 3 pontos $(0=$ nunca cochilar até $3=$ alta chance de cochilar), com o escore máximo de 24 , que representa alto nível de sonolência. O intervalo proposto para propensão de sono normal é de 0 a $10 .{ }^{13}$

O The Medical Study 36-item Short- Form Health Survey (SF-36) foi utilizado para avaliar a qualidade de vida. Este questionário multidimensional é formado por 36 itens, os quais são englobados oito domínios ou componentes que avaliam: a capacidade funcional, aspectos físicos, dor, estado geral de saúde, vitalidade, aspectos sociais, aspectos emocionais, saúde mental e uma questão da avaliação comparativa entre as condições de saúde atual e a que antecede um ano da entrevista. Para a interpretação dos resultados é dado um escore para cada questão, que posteriormente são transformados numa escala de zero a 100, onde zero corresponde ao "pior estado de saúde" e cem ao "melhor estado de saúde". Esse instrumento possui propriedades psicométricas de confiabilidade e validade documentada na literatura nacional e internacional. ${ }^{14}$

O Teste de Caminhada de Seis minutos (TC6) foi utilizado para avaliação da capacidade funcional e aplicado de acordo com as recomendações da American Thoracic Society (ATS). ${ }^{15}$ O TC6 foi realizado em um corredor de 25 metros e aplicado por um único avaliador. A frequência respiratória, a frequência cardíaca e a $\mathrm{SpO}_{2}$ foram avaliadas antes e após o TC6. A percepção do esforço ao exercício foi avaliada pela Escala de Borg ${ }^{16}$ ao final do TC6. A $\mathrm{SpO}_{2}$ foi avaliada pela oxímetria de pulso (MD300C1, Choice Medical, Solmedica, Porto Alegre, RS, Brasil).

A função pulmonar foi avaliada pela espirometria através de um espirômetro portátil (Spirobank II-Medical International Research, Waukesha, WI, EUA), com o objetivo de obter os valores preditos. ${ }^{17}$ Para a realização do teste, os indivíduos permaneceram sentados confortavelmente, e foram orientados a repousarem por mais de dez minutos antes do teste. Durante este período, os procedimentos a serem realizados foram descritos cuidadosamente. Enfatizou-se a necessidade de se evitar vazamentos em torno da peça bucal e a utilização do clip nasal, solicitou-se que o sujeito realizasse uma inspiração máxima, seguida de expiração máxima e sustentada através do bucal do aparelho, até ser ordenado a interromper a manobra. Conforme recomendado pela ATS e European Respiratory Society (2005) e baseado nos critérios de reprodutibilidade e aceitabilidade, foram realizadas três manobras (variabilidade $<5 \%$ ) e considerada a melhor curva para o estudo. As variáveis analisadas foram, a capacidade vital forçada (CVF), o volume expiratório forçado no primeiro segundo (VEF1), a relação VEF1/ CVF, o pico de fluxo expiratório (PFE), o fluxo expiratório forçado entre 25 e $75 \%$ da curva de CVF (FEF25-75) e a ventilação voluntária máxima (VVM). Para a medida da VVM o individuo foi instruído a inspirar e expirar repetidamente pelo bucal com maior esforço possível, durante 10 segundos. Os valores foram registrados em unidades de medida absolutas e em percentuais do predito.

A força muscular respiratória foi mensurada por manovacuômetro digital (MVD 300, Microhard Sistema, Globalmed, Porto Alegre, RS, Brasil), estando o sujeito confortavelmente sentado utilizando um clip nasal. Para a determinação da força muscular inspiratória foi mensurado a pressão inspiratória máxima (PImax) obtida com a manobra de inspiração máxima forçada a partir do volume residual. A força muscular expiratória foi avaliada através da pressão expiratória máxima (PEmax), com uma expiração máxima forçada a partir da capacidade pulmonar total (CPT). As manobras foram realizadas seis vezes, com intervalo de um minuto entre elas. Os maiores valores de PImax e PEmax foram registrados, desde que, a diferença entres os dois maiores valores fosse menor do que $10 \% .{ }^{18}$

Para a análise estatística foi utilizado o programa Statistical Package for the Social Sciences (SPSS; versão 13.0). Os dados estão apresentados em média e desvio-padrão (DP). A normalidade dos dados foi verificada pelo teste de KolmogorovSmirnov. Diante da normalidade na distribuição dos dados em todas as variáveis, a análise comparativa entre os grupos foi realizada pelo teste t de Student para amostras independentes. A taxa de erro alfa de $5 \%(p<0,05)$ foi considerada significante.

\section{Resultados}

A caracterização dos sujeitos encontra-se na Tabela 1. Os GC e GAOS não apresentaram diferen- 
ça em relação à idade, IMC e demais variáveis antropométricas na admissão ao estudo. Como esperado, o GAOS apresentou maiores valores no PSQIBR e na ESS.

A Tabela 2 apresenta os resultados referentes ao comportamento das variáveis analisadas durante o TC6. Os pacientes do GAOS percorreram uma distância de $12 \%$ menor que os voluntários do grupo controle.

As variáveis relacionadas à força muscular respiratória e função pulmonar não apresentaram diferenças entre os grupos para os valores absolutos e percentuais do predito. Os dados são apresentados na Tabela 3.

Tabela 1. Caracterização do grupo controle (GC) e do grupo apneia obstrutiva do sono (GAOS).

\begin{tabular}{lrrr}
\hline Variável & GC & GAOS & Valor de p \\
\hline Idade (anos) & $49,7 \pm 9,4$ & $49,7 \pm 8,8$ & 0,999 \\
Índice de massa corporal $\left(\mathrm{kg} / \mathrm{m}^{2}\right)$ & $27,0 \pm 3,1$ & $27,2 \pm 2,5$ & 0,835 \\
Pressão arterial sistólica $(\mathrm{mmHg})$ & $130,0 \pm 12,5$ & $130,0 \pm 13,3$ & 0,999 \\
Pressão arterial diastólica $(\mathrm{mmHg})$ & $84,2 \pm 12,6$ & $86,8 \pm 9,5$ & 0,972 \\
Saturação periférica de oxigênio (\%) & $98,1 \pm 1,4$ & $98,1 \pm 0,7$ & 0,070 \\
Frequência cardíaca (bpm) & $70,0 \pm 8,5$ & $76,2 \pm 11,8$ & 0,187 \\
Frequência respiratória $(\mathrm{rpm})$ & $16,3 \pm 3,0$ & $15,1 \pm 2,6$ & 0,977 \\
Circunferência da cintura (cm) & $91,2 \pm 9,7$ & $91,0 \pm 21,6$ & 0,177 \\
Circunferência de quadril $(\mathrm{cm})$ & $99,5 \pm 8,8$ & $102,8 \pm 5,4$ & 0,565 \\
Relação cintura quadril $(\mathrm{cm})$ & $0,92 \pm 0,1$ & $0,89 \pm 0,2$ & 0,081 \\
Circunferência abdominal $(\mathrm{cm})$ & $96,4 \pm 9,2$ & $101,5 \pm 8,5$ & 0,479 \\
Circunferência do pescoço $(\mathrm{cm})$ & $38,4 \pm 3,8$ & $39,3 \pm 3,3$ & 0,000 \\
Pittsburg & $4,6 \pm 3,3$ & $22,3 \pm 8,7$ & 0,002 \\
Epworth & $7,2 \pm 3,9$ & $12,5 \pm 5,7$ &
\end{tabular}

Resultados expressos em média e desvio padrão ( $(\mathrm{DP})$; Teste "t" para amostras independentes; nível de significância $p<0,05$.

Tabela 2. Distância percorrida e monitorização durante o TC6 no grupo controle (GC) e no grupo apneia obstrutiva do sono (GAOS).

\begin{tabular}{lrrr} 
Variável & GC & GAOS & Valor de p \\
\hline Distância no TC6 (m) & $538,8 \pm 83,3$ & $475,6 \pm 89,5$ & 0,033 \\
Frequência cardíaca inicial (bpm) & $73,8 \pm 14,2$ & $71,2 \pm 10,0$ & 0,504 \\
Frequência cardíaca final (bpm) & $97,2 \pm 19,5$ & $98,3 \pm 18,7$ & 0,853 \\
Pressão arterial sistólica inicial (mmHg) & $129,4 \pm 12,6$ & $132,6 \pm 18,1$ & 0,541 \\
Pressão arterial sistólica final (mmHg) & $136,7 \pm 14,1$ & $148,4 \pm 21,2$ & 0,056 \\
Pressão arterial diastólica inicial (mmHg) & $85,0 \pm 12,0$ & $88,4 \pm 11,7$ & 0,386 \\
Pressão arterial diastólica final (mmHg) & $82,8 \pm 22,7$ & $96,3 \pm 17,4$ & 0,049 \\
Frequência respiratória inicial (rpm) & $16,1 \pm 2,5$ & $21,1 \pm 3,6$ & 0,004 \\
Frequência respiratória final (rpm) & $19,7 \pm 3,0$ & $97,7 \pm 1,0$ & 0,196 \\
Saturação periférica de oxigênio inicial (\%) & $97,8 \pm 1,4$ & $96,6 \pm 3,6$ & 0,201 \\
Saturação periférica de oxigênio final (\%) & $97,8 \pm 1,6$ & & 0,6 \\
\hline
\end{tabular}

TC6 = Teste de caminhada de seis minutos. Resultados expressos em média e desvio padrão ( \pm DP); Teste “t” para amostras independentes; nível de significância $p<0,05$. 
Tabela 3. Força muscular respiratória e função pulmonar do grupo controle (GC) e do grupo apneia obstrutiva do sono (GAOS).

\begin{tabular}{lrrr}
\hline Variável & GC & GAOS & Valor de p \\
\hline Pressão inspiratória máxima $\left(\mathrm{cmH}_{2} \mathrm{O}\right)$ & $111,7 \pm 28,7$ & $107,2 \pm 26,1$ & 0,614 \\
Pressão expiratória máxima $\left(\mathrm{cmH}_{2} \mathrm{O}\right)$ & $138,5 \pm 31,1$ & $151,4 \pm 39,0$ & 0,265 \\
Capacidade vital forçada (L) & $4,0 \pm 1,1$ & $4,6 \pm 1,4$ & 0,161 \\
Capacidade vital forçada (\%pred) & $102,2 \pm 12,3$ & $110,1 \pm 12,5$ & 0,065 \\
VEF1(L) & $3,3 \pm 0,9$ & $3,6 \pm 0,9$ & 0,304 \\
VEF1 (\%pred) & $103,1 \pm 12,6$ & $110,0 \pm 12,5$ & 0,096 \\
VEF1/CFV (L) & $82,7 \pm 6,6$ & $80,1 \pm 6,7$ & 0,233 \\
VEF1/CFV (\%pred) & $104,7 \pm 9,0$ & $101,9 \pm 8,8$ & 0,343 \\
Pico de fluxo expiratório (L/S) & $7,3 \pm 2,1$ & $8,4 \pm 2,4$ & 0,124 \\
Pico de fluxo expiratório (\%pred) & $97,9 \pm 22,3$ & $103,9 \pm 24,0$ & 0,430 \\
FEF25-75 (L/S) & $3,7 \pm 1,1$ & $3,6 \pm 0,8$ & 0,637 \\
FEF25-75 (\%pred) & $100,2 \pm 24,7$ & $96,8 \pm 23,0$ & 0,661 \\
Ventilação voluntária máxima (L) & $134,4 \pm 28,8$ & $141,0 \pm 31,5$ & 0,508 \\
Ventilação voluntária máxima (\%pred) & $109,7 \pm 13,4$ & $116,4 \pm 14,2$ & 0,144
\end{tabular}

VEF1 = Volume expiratório forçado no primeiro segundo; FEF25-75: Fluxo expiratório forçado entre 25 e $75 \%$ da curva de CFV; (\% pred): percentual do predito. Resultados expressos em média e desvio padrão ( $\pm D P)$; Teste "t" para amostras independentes; nível de significância $p<0,05$.

A Figura 1 apresenta os resultados referentes à qualidade de vida dos sujeitos participantes do estudo. A qualidade de vida do GAOS é inferior, observada pelos menores valores nos domínios de capacidade funcional, estado geral de saú- de e saúde mental. Dentre os domínios do SF-36, o GC apresentou um aumento de 10,6 \% na capacidade funcional, de $15,6 \%$ no estado geral de saúde e de $16,3 \%$ na saúde mental em comparação ao GAOS.

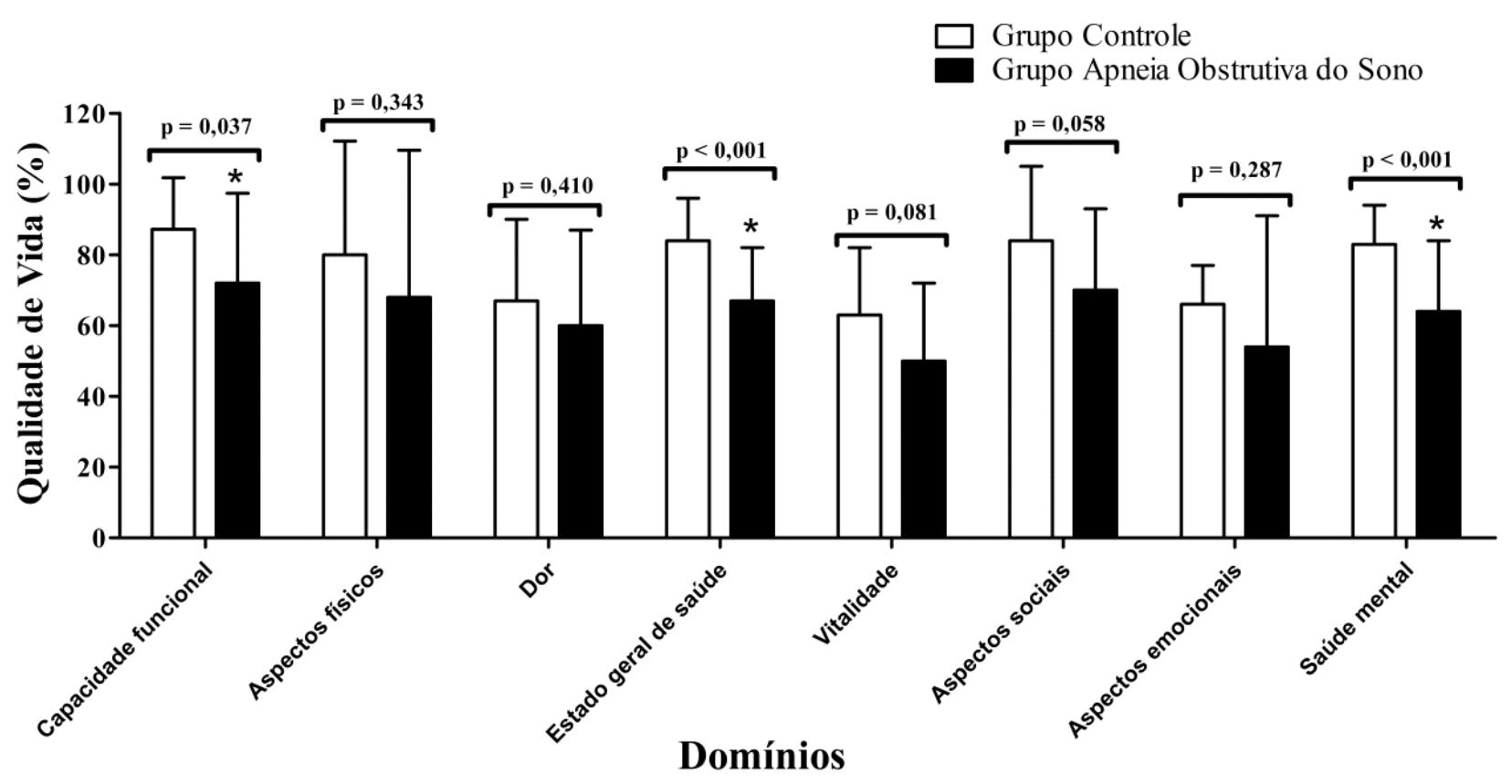

Figura 1. Valores referentes à qualidade de vida em todos os domínios do SF-36 em ambos os grupos.

Resultados expressos em média e desvio padrão ( $\pm \mathrm{DP})$; Teste "t" para amostras independentes;

*: diferença significativa entre os grupos. 


\section{Discussão}

Recentes estudos $3,4,5$ têm avaliado e indicado as principais complicações causadas pela AOS. Assim como em nossa investigação, alguns relatos indicam um prejuízo na qualidade de vida e na capacidade funcional dessa população. ${ }^{3}$ Como esperado, o presente estudo demonstrou redução na qualidade do sono e aumento no nível de sonolência ao longo do dia nos pacientes com apneia obstrutiva do sono. Entretanto, nossos resultados demonstram que esses pacientes não apresentam comprometimento da função pulmonar e da força da musculatura respiratória.

Estudo prévio, que avaliou a sonolência excessiva diurna de chineses com diagnóstico de apneia obstrutiva do sono, demonstrou uma maior pontuação no ESS nesses pacientes, concluindo que o índice de sonolência é maior nesta condição, ${ }^{19}$ o que corrobora com os nossos achados.

Os nossos achados apontam a piora significativa em três domínios do SF-36 (capacidade funcional, estado geral de saúde e saúde mental) no GAOS. Esses resultados são confirmados por estudos prévios. 3,20,21 Em estudo multicêntrico foi demonstrado que pacientes com AOS apresentam piores escores em todos os domínios da qualidade de vida. ${ }^{3}$ Outros achados ${ }^{20}$ confirmam estes resultados, como um estudo onde também foi avaliada a qualidade de vida em pacientes com AOS e observou-se que todos os domínios foram prejudicados nesta população. ${ }^{20}$ Num terceiro estudo observouse uma piora em seis dos oitos domínios da escala SF-36 em pacientes com AOS. ${ }^{21}$ A piora nos índices de qualidade de vida em pacientes com diagnóstico de AOS está relacionada à sonolência excessiva, à obesidade, à fragmentação do sono e à hipoxemia. ${ }^{3}$ Em comparação com indivíduos saudáveis, os pacientes com AOS são menos propensos a se envolver em atividades sociais, além de apresentarem maiores limitações físicas e emocionais. ${ }^{3}$

Esses comprometimentos dos domínios do SF36 , em especial com relação à vitalidade, aspectos sociais, aspectos emocionais, saúde mental e capacidade funcional, demonstram que pessoas com algum comprometimento de sua saúde sentem-se desmotivados, apresentam falta de concentração, o que pode causar limitações físicas. ${ }^{22}$ Além disso, os menores valores nestes domínios sugerem que a apneia obstrutiva do sono está associada à fadiga e a dificuldade de concentração ou perda de memória. ${ }^{23}$ Entretanto, Gebocka e Colaboradores $(2006)^{22}$ não observaram quaisquer perturbações psicológicas e comprometimento da qualidade de vida nesses pacientes.

A capacidade funcional avaliada pela distância percorrida no TC6 apresentou uma redução de $12 \%$ nos pacientes com AOS. As possíveis razões para esta redução são multifatoriais e representam uma associação entre o sedentarismo, a obesidade, as doenças cardiovasculares, dispneia e outros mecanismos ainda desconhecidos. ${ }^{24}$ Ressalta-se que algumas destas variáveis (obesidade e doenças cardiovasculares) foram controladas em nosso estudo, pois fizeram parte dos critérios de exclusão. Portanto, não seriam estes os fatores de exposição capazes de explicar tal alteração.

Evidências prévias ${ }^{25,26}$ demonstram que o comprometimento da capacidade funcional na população em geral, incluindo pacientes com AOS, está relacionado a hábitos sedentários cada vez mais prevalentes em adultos. ${ }^{27}$ Tal comprometimento favorece o aumento do risco do aparecimento de doenças e agravos crônicos que incluem problemas cardiovasculares, diabetes, osteoporose, obesidade, dislipidemias e depressão. Baseando-se nestas afirmações, bons níveis de capacidade funcional podem trazer benefícios para a saúde e contribuir para uma melhor qualidade de vida. ${ }^{27}$

Os resultados relacionados à função pulmonar e força muscular respiratória, não diferiram entre os pacientes e controles. A ausência de alteração na função pulmonar e força muscular respiratória é algo esperado nesta população, visto que a fisiopatologia da AOS relaciona-se à disfunção de vias aéreas superiores, causada, entre outros, pelo aumento da complacência da musculatura faríngea e pelo acúmulo de gordura parafaríngea, que estão comumente associados ao colapso das vias aéreas superiores. ${ }^{28}$

$\mathrm{Na}$ literatura atual, poucos são os estudos encontrados que avaliam a função pulmonar em pacientes com AOS, sendo encontrados apenas estudos que avaliam esta variável após uso de CPAP. Estudo prévio 24 mostrou uma melhora da função pulmonar após o uso do CPAP, mas não mencionou um comparativo da função pulmonar entre os pacientes com apneia obstrutiva do sono e indivíduos 
saudáveis, assim como não avaliam a força muscular respiratória nesses pacientes.

Portanto, a AOS por ser descrita como uma condição comum causada por colapso intermitente das vias aéreas superiores durante o sono, ${ }^{2}$ nos instiga a, cada vez mais, refletir sobre a recuperação da capacidade funcional, que por sua vez, proporcionará uma melhor perspectiva de qualidade de vida desses pacientes. Dentre as limitações deste estudo, não foram avaliadas as atividades de vida diária, o nível de funcionalidade e a aptidão cardiorrespiratória, o que poderia fornecer uma estimativa mais abrangente da capacidade funcional destes pacientes. As medidas de função pulmonar pela pletismografia e a avaliação da resistência muscular respiratória representam metas para ampliar a análise das condições respiratórias em futuros estudos.

\section{Conclusão}

Nossos resultados demonstraram que pacientes com apneia obstrutiva do sono apresentam redução na capacidade funcional e na qualidade de vida, em comparação a sujeitos saudáveis. Estas alterações podem indicar a necessidade de estratégias de intervenção voltadas ao aprimoramento da capacidade funcional nesta população. O prejuízo na qualidade de vida, identificado nos domínios capacidade funcional, estado geral de saúde e saúde mental, demonstram algumas particularidades e poderão nortear as ações interdisciplinares no manejo da AOS. Houve similaridade na função pulmonar e na força muscular respiratória entre o grupo de pacientes e os sujeitos saudáveis.

\section{Referências}

1. Davatz AF, Andrade HLI, Melo IVP, Alve TK, Aleva NA, Vitela JD et al. Síndromes da apneia e hipopnéia obstrutiva do sono: revisão da literatura. Revista Eletrônica Acervo Saúde 2012; 1: 27-33.

2. Cintra FD, Tufik S, Paola A, Feres MC, Melo-Fujita L, Oliveira $W$ et al. Cardiovascular profile in patients with obstructive sleep apnea. Arq Bras Cardiol. 2011; 96: 293-9.

3. Diaferia G, Badke L, Santos-Silva R, Bommarito S, Tufik S, Bittencourt $L$. Effect of speech therapy as adjunct treatment to continuous positive airway pressure on the quality of life of patients with obstructive sleep apnea. Sleep Med. 2013; 14: 628-35.

4. Gurubhagavatula I. Consequences of obstructive sleep apnea. Indian J Med Res. 2010; 131:188-95.
5. Dutt N, Janmeja AK, Mohapatra PR, Singh AK. Quality of life in patients with syndrome of obstructive sleep apnea and its relations hip with disease severity. Lung Índia. 2013; 30: 28994.

6. Kocks JW, Asijee GM, Tsiligianni LG, Kerstjens HA, Van der Molen T. Functional status measurement in COPD: a review of available methods and their feasibility in primary care. Prim Care Respir J. 2011; 20:269-75.

7. Shah N, Roux F, Mohsenin V. Improving Health-Related quality of life in patients with obstructive sleep apnea: What are the available options? Treat Respir Med. 2006; 5: 235-44.

8. Lopes C, Esteves AM, Bittencourt LR, Tufik S, Mello MT. Relationship between quality of life and severity of obstructive sleep apnea syndrome. Braz J Med Biol Res. 2008; 41: 90813.

9. Butner KL, Hargens TA, Kaleth AS, Miller LE, Herbert WG. Association of obstructive sleep apnea severity with exercise capacity and health-related quality of life. Am J Med Sci. 2013; 5: 362-6.

10. Heinzer R, Branco DP, Malhotra, A, L lo Y, Dover L, Stevenson $\mathrm{KE}$ et al. Effect of expiratory positive airway pressure on sleep disordered breathing. Sleep. 2008; 31: 429-32.

11. Jordan AS, Mcsharry DG, Malhotra A. Adult obstructive sleep apnea. Lancet. 2014; 383: 736-47.

12. Bertolazi AN. Tradução, adaptação cultural e validação de dois instrumentos de avaliação do sono: escala de sonolência de Epworth e índice da qualidade do sono de Pittsburg. Porto Alegre: Faculdade de Medicina, Universidade Federal do Rio Grande do Sul, 2008.

13. Johns MW. Sensitivity and specificity of the multiple sleep latency test (MSLT), the maintenance of wakefulness test and the Epworth sleepiness scale: failure of the MSLT as a gold standard. J Sleep Res. 2000; 9: 5-11.

14. Ekman I, Fagerberg B, Lundman B. Health-related quality of life and sense of coherence among elderly patients with severe chronic heart failure in comparison with healthy controls. Heart Lung. 2002; 31: 94-101.

15. Kohl LM, Signori LU, Ribeiro R, Silva AMV, Moreira PR, Dipp T, Sbruzzi G, Plentz RDM. Prognostic value of six-minute walk test in end-stage renal disease life expectancy prospective cohort study clinics. Rev USP (Impr.).2012; 67 : 581-6.

16. Dorneles $S Q$, Signori LU, Corrêa LQ, Silveira DF, Guerreiro LF, Teixeira AO. Efeitos da hospitalização na capacidade funcional e na qualidade de vida de pacientes cardiometabólicos. Conscientiae saúde. 2014; 13: 45-72.

17. Pereira CAP, Barreto SP, Simöes JG, Pereira FWL, Gerstler JG, Nakatani J. Reference Values for Spirometry in Brazilian Adults. J Bras Pneumol. 1992; 18: 10-22.

18. Kaminski DM, Schaan BD, Silva AM, Soares PP, Plentz RD, Dall'ago P. Inspiratory muscle weakness is associated with autonomic cardiovascular dysfunction in patients with type 2 diabetes mellitus. Clin Auton Res. 2011; 21:29-35.

19. Chen R, Xiong KP, Lian YX, Huang JV, Zhao MV, Li JL, Liu CF. Daytime sleepiness and its determining factors in Chinese obstructive sleep apnea patients. Sleep Breath. 2011; 15:129-35

20. D'ambrosio C, Bowman T, Mohsenin V. Quality of life in patients with obstructive sleep apnea: Effect of nasal continuous positive airway pressure-a prospective study. Chest. 1999; $115: 123-9$ 
21. Akashiba T, Kawahara S, Akahoshi T, Omori C, Saito O, Majima $T$ et al. Relationship between quality of life and mood or depression in patients with obstructive severe sleep apnea syndrome. Chest. 2002; 122: 861-65.

22. Gebocka A, Kossowska A, Bednarek M. Obstructive sleep apnea and quality of life. J Physiol Pharmacol. 2006; 57:11117.

23. Hyun Uk J, Ki SP, Sang MC, Ho WL, Sung WK, Seung HL et al. Development of the Korean version of Sleep Apnea Quality of Life. Clin Exp Otorhinolaryngol. 2014; 7:24-9.

24. Rizzi CF, Cintra F, Risso T, Pulz C, Tufik S, De paola A, Poyares D. Exercise capacity and obstructive sleep apnea in lean subjects. Chest. 2011; 137: 9-14.

25. Guillermo LQ, Gal TJ, Mair EA. Obstructive sleep apnea affects aerobic fitness? Ann Otol Rhinol Laryngol. 2006; 115:715-20.

26. Mansukhani MP, Alisson TG, Jimenez FL, Somers VK, Caples SM. Functional aerobic capacity in patients with sleep-disordered breathing. Am J Cardiol. 2014; 111:1650-4.

27. Corseuil MW, Petroski EL. Baixos níveis de aptidão física relacionada à saúde em universitários. Rev Bras Educ Fís Esporte. 2010; 24: 49-54.

28. Tufik S, Santos-Silva R, Taddei JA, Bittencourt LRA. Obstructive Sleep Apnea Syndrome in the São Paulo Epidemiologic Sleep Study. Sleep Med. 2010 ; 11: 441- 4. 